\title{
Improving Social Game Engagement on Facebook through Enhanced Socio-Contextual Information
}

\author{
Ben Kirman ${ }^{1}$, Shaun Lawson ${ }^{1}$, Conor Linehan ${ }^{1}$, Francesco Martino ${ }^{2}$, Luciano Gamberini ${ }^{2}$ and \\ Andrea Gaggioli ${ }^{3}$ \\ ${ }^{1}$ Lincoln Social Computing \\ ${ }^{2}$ Human Technology Lab \\ Research Centre (LiSC) \\ University of Lincoln \\ Lincoln, UK \\ Department of General Psychology \\ ${ }^{3}$ Applied Technology for \\ University of Padova, Padova, Italy \\ Neuro-Psychology Lab, \\ Istituto Auxologico Italiano, \\ Milan, Italy \\ \{bkirman, slawson, clinehan\}@lincoln.ac.uk; \{francesco.martino,luciano.gamberini\}@unipd.it; \\ andrea.gaggioli@auxologico.it
}

\begin{abstract}
In this paper we describe the results of a controlled study of a social game, Magpies, which was built on the Facebook Online Social Network (OSN) and enhanced with contextual social information in the form of a variety of social network indices. Through comparison with a concurrent control trial using an identical game without the enhanced social information, it was shown that the additional contextual data increased the frequency of social activity between players engaged in the game. Despite this increase in activity, there was little increase in growth of the player-base when compared to the control condition. These findings corroborate previous work that showed how socio-contextual enhancement can increase performance on task-driven games, whilst also suggesting that it can increase activity and engagement when provided as context for non task-driven game environments.
\end{abstract}

\section{Author Keywords}

Social Network Analysis, Social Context, Social Gaming, Social Visualisations, Mediated Interaction

\section{INTRODUCTION}

The notion of Social Presence has long been a popular topic for research in the field of HCI [1]. Researchers in Computer-mediated communication (CMC) have conducted a great deal of work on replicating typical non-verbal aspects of face-to-face communication in computer mediated social environments and also introducing new non-verbal cues in a manner that is respectful of the context in which these interactions take place [3]. One of the major strategies for increasing the richness of social presence has been by exposing the underlying social behaviours of group members, and making implicit factors in computermediated communication explicit. For instance, applications have been created that expose social network information [10] as "Socio-Contextual" information (data that makes hidden information about the social context of individuals more visible) to participants within group working scenarios [5,2,8] and games [6].
In related work to that described here, Martino et al found that displaying socio-contextual information in the form of Social Network Analysis (SNA) indices to players of a collaborative group treasure hunting game had a positive effect on increasing group ability to cooperate and engage with the game [9].

\section{OSNs as Socially Enhanced Application Platforms}

The explosive growth in popularity of Online Social Networks (OSNs) such as MySpace and Facebook serves to highlight the value of rich social context in mediated environments. Such sites act as a social management tool through which users can describe their various social relationships. Upon this Social Graph, mediated applications can be built that take advantage of the social context provided in order to create more engaging experiences [4,11]. These applications range in complexity from simple messaging and status-sharing tools, to complex and sophisticated games involving rich social data. For example the game Restaurant City allows players to "employ" real friends as waiters and chefs in their virtual restaurants while FriendFolio allows players to buy and sell their own friends in popularity based stock market.

The combination of research on socio-contextual augmentation and the emerging importance of OSNs as a platform for social gaming led us to seek to investigate whether this same augmentation is effective in applications built on existing social network structures (e.g. Facebook) and whether this would extend to activities not centred around task completion or problem solving. 


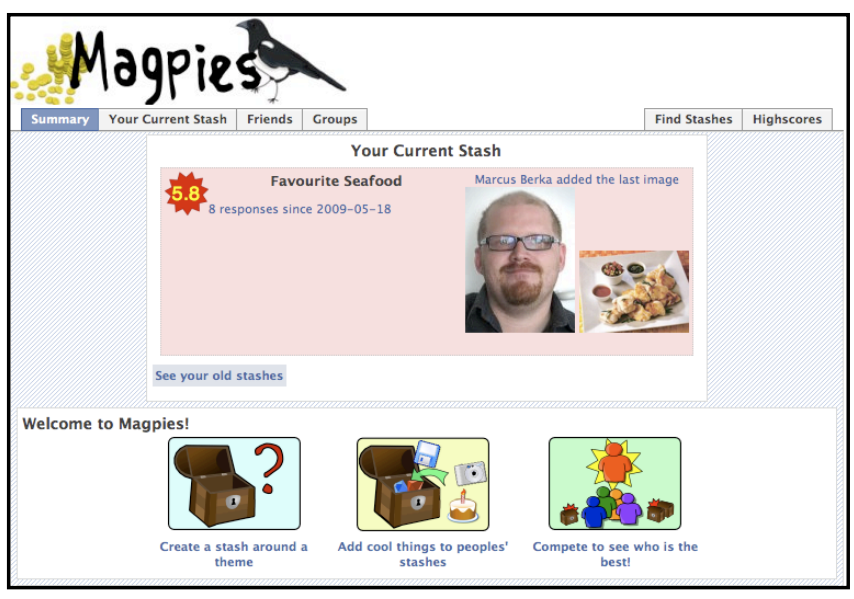

Figure 1 - Magpies Interface

\section{MAGPIES}

In order to answer these research questions, a social game application was created and deployed using the Facebook platform. The platform exposes large amounts of information about the social graph to application developers through an API to allow the creation of socially rich games and tools. The game described here, called Magpies, is centred on the theme of collection; in many cultures, Magpies are birds associated with the activity of collecting small shiny trinkets with which they decorate their nests. Players in the game work individually and create collections or stashes around a theme determined through free text entry. Other players are invited to contribute items that match the theme, in the form of text, images and/or locations. The stash then builds up over time as a list of curiosities that match this theme. Players can rate one another's stashes and compete to build the most popular collections.

Players are also able to create and join arbitrary groups within the system. Groups are ranked against each other based on the status of the players within, to add a socially competitive angle to an otherwise individually driven game.

Magpies was tested internally through a series of usability evaluations and focus groups in order to fine tune the game design and interface, before being released officially on the Facebook platform and being made open to the public.

\section{Social Context Information}

In Magpies, as an addition to the main game, users were exposed to a variety of measures based on a selection of standard Social Network Analysis (SNA) indices (e.g. as in [14]). These were split into group and individual indices. The Group Indices included Group Centralisation, Group Reciprocity and Group Density. The Individual Indices included Degree Centrality, In-Degree Centrality, OutDegree Centrality and Reciprocity.
These measures were calculated regularly based on activity within the game, and presented to the user as values between zero and five "stars" (as seen in Figure 2). The technical names for the indices were replaced in the interface by labels validated by focus groups as more meaningful to players (i.e. "Reciprocity" became "Equality") and supported with descriptions and examples within the game.

Additional visualisations allowed players to see activity in the form of network graphs. For example, it was possible to view the pattern of interactions between players within groups in the form of a network graph, where individuals were represented by nodes and connected by arrows. These arrows represent the act of one player contributing to the stash of another. Players could also visualise group membership by seeing a graph that represented each group as a node connected by edges indicating shared membership. This aided the players when choosing to join groups.

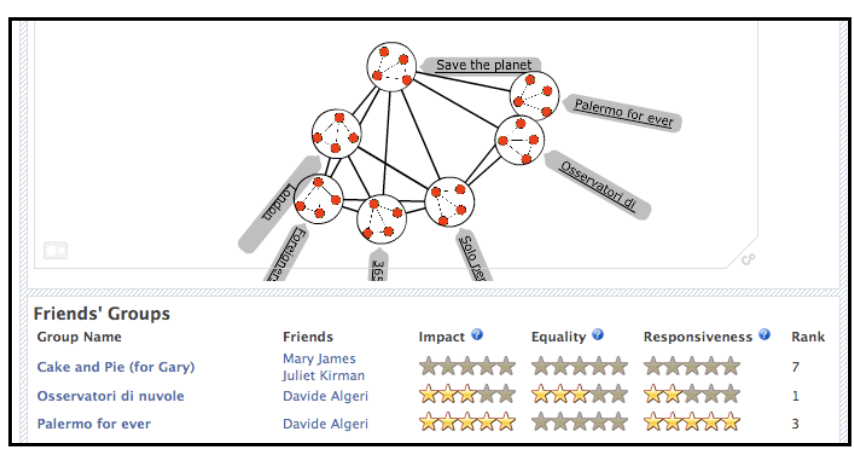

Figure 2 - Group Social Network Indices in Magpies

It must be emphasised that the enhanced social contextual information provided to players in the Magpies condition was provided as additional supporting information around the core mechanics of the game. There was no central task players were asked to perform with the aid of this information, and no problems that required solving through organised group collaboration.

\section{METHOD}

In order to conduct a controlled experiment, a second, almost exact duplicate of the Magpies game was created called Beachcombers, so-named from the activity of exploring beaches to discover trinkets and treasures washed ashore by the tides. This game was identical to Magpies apart from the branding and the fact that the social contextual information was hidden from view of the players themselves. 


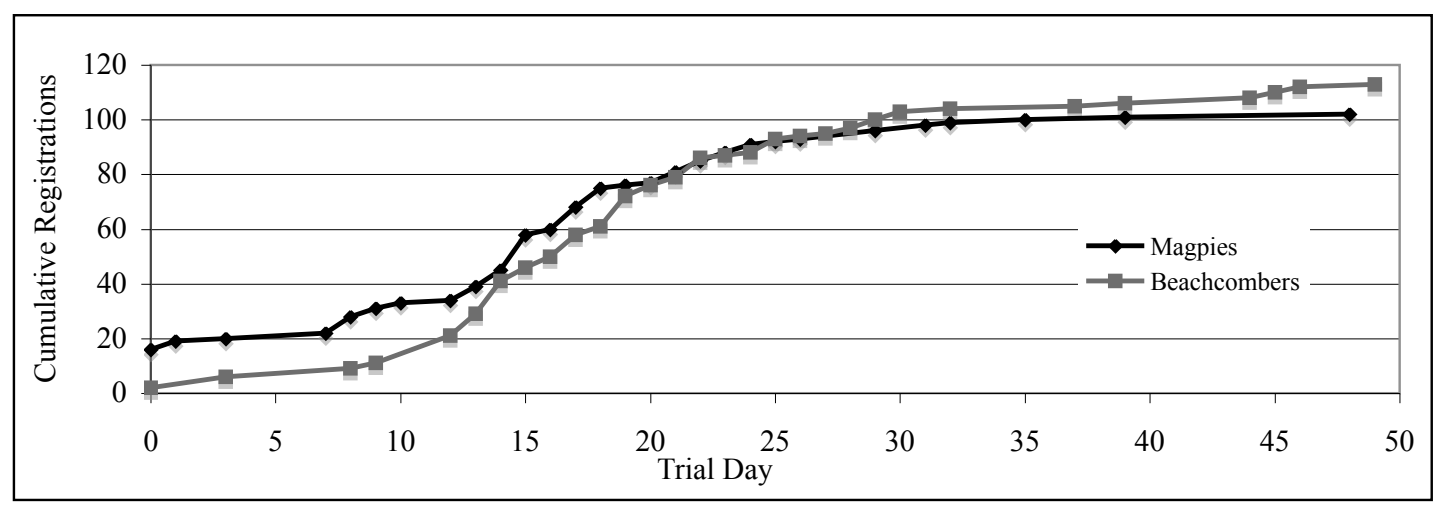

Figure 3 - Cumulative Registrations for both Conditions

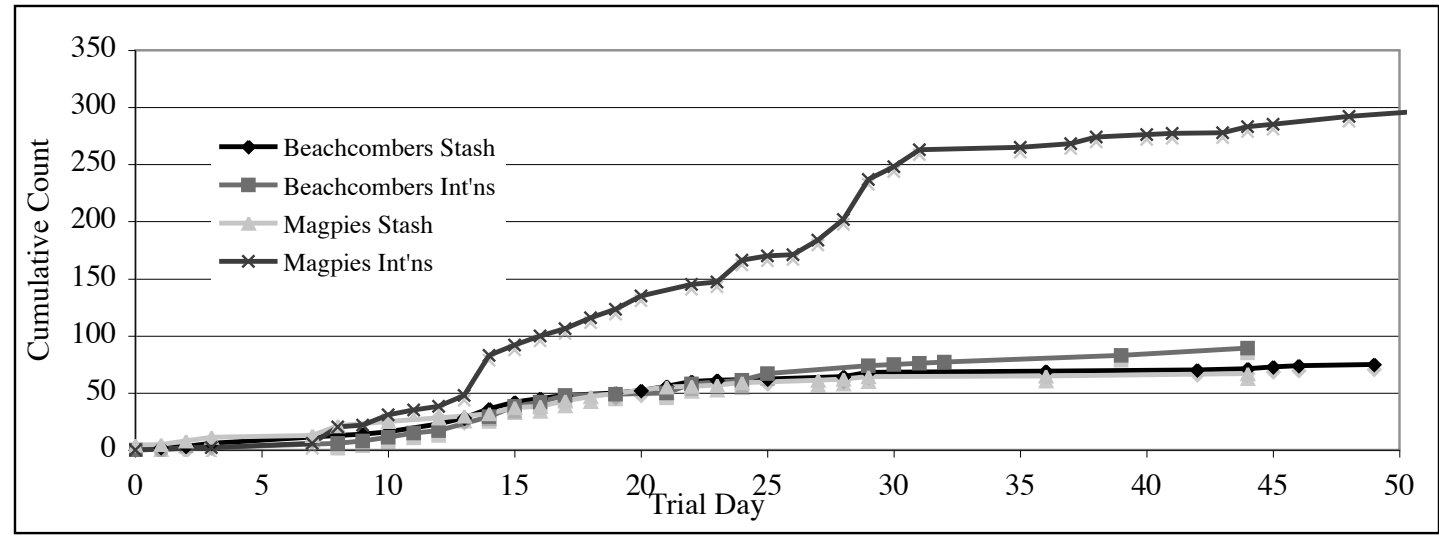

Figure 4 - Interactions and Stashes in both Conditions

Both games were released at the same time to a small group of volunteer participants selected by trial organisers and from then additional players were free to join the game through natural "viral" spread. Each new player was asked to complete a release form for trial participation before beginning to play. All interactions with both versions of the system, including page access and usage of functionality, were recorded, along with the results of the social network indices, for post-trial analysis. Players were able to join either game freely. Despite this only one (non-invited) participant interacted with both games during the trial period.

The concurrent trials for both games lasted for 50 days starting at the end of April 2009. At the end of the trial period questionnaires were distributed to all participants in order to gather additional non-procedural information about the trial.

\section{RESULTS}

Over the course of the trial a total of 215 players played the two games. Between both conditions 166 collections were created, and these provoked a total of 364 response interactions (including a combination of text, images and locations) from other players.
Table 1 - Collections and Interactions in both Conditions

\begin{tabular}{l|lll}
\hline & Players (N) & Collections & $\begin{array}{l}\text { Total } \\
\text { Interactions }\end{array}$ \\
\hline Magpies & 102 & 67 & 297 \\
$\begin{array}{l}\text { Beachcombers } \\
\text { (Control) }\end{array}$ & 113 & 75 & 89 \\
\hline
\end{tabular}

Table 1 shows the breakdown of players, collections and response interactions between the two distinct conditions. Both conditions had a similar number of players who created a similar number of collections. There is a striking difference in the number of interactions contributed by players in the augmented (Magpies) condition.

\section{Growth of Player-base}

Figure 3 shows the cumulative growth in registrations experienced by each application during the trial period. The graphs both follow a very similar "S-curve" of diffusion [12]. A month into the trial, the rate of new registrations slowed. This pattern of growth matches the experience of Facebook applications in general [4,11]. Both Magpies and Beachcombers experienced extremely similar patterns of growth, which indicates that the additional socio-contextual information in the augmented condition does not appear to be an interesting factor for attracting new players, or 
motivating existing players to actively engage in recruitment.

\section{Effect of Enhanced Social Context on Interactions}

In-game collections created in the socio-contextually augmented condition (Magpies) provoked many more response interactions from other players than those collections in the non-augmented condition.

Figure 4 shows the pattern of interactions (i.e. the act of a player making a contribution to another player's collection within the game) experienced in both conditions over time, compared with the growth of collection creation. As can be clearly observed, the pattern of interactions in the augmented condition appears to be highly accelerated when compared with the non-augmented condition, despite the patterns of collection creation and player-base growth being very similar. This indicates an increase in engagement with the game for the players in the augmented condition.

\section{CONCLUSION}

In this paper we have described a new application, Magpies, which is a non-task-oriented social game deployed on the Facebook OSN. Magpies uses social network analysis (SNA) information to add social data to the context of a player's interactions within the game.

In a controlled study involving over two hundred users, the research shows that when players were exposed to this additional socio-contextual information, they were more likely to be involved in a higher number of social interactions with other game players compared with a game that did not display this socio-contextual information.

These results corroborate findings in previous work by Martino et al [9], in which players exposed to SNA information while playing games demonstrated increased activity and engagement, and extends this work further by showing that the observed increase in activity is not just limited to games centred around collaborative task completion or problem solving, but also applies to games such as Magpies, where the central objective is not as clearly defined and the additional social information is provided simply as context to the game play.

Magpies was deployed on the social platform of Facebook and allowed to grow naturally through the social graph. However, results show that the use of socio-contextual augmentation did not have any significant effect on the rate of growth of the player-base. The increased engagement and increased social presence observed with active players does not appear to be a motivating factor for new users to join the game. Since the socio-contextual enhancement in the Magpies condition was not related to the act of recruitment it may not have impacted on recruiting behaviours. In terms of Kluger \& DeNisi's theory of Feedback Intervention [7], when goals and feedbacks are clear as in our socio-contextual information, optimal usage of the feedback occurs, and users' attention is oriented toward task motivation level (in which users usually try to find a way to fill the gap between feedback value and goal value). A feedback on activity (such as the one we are proposing) may shift users' attention toward taskmotivation level and therefore drive users' attention toward increasing social interactions (indices values), since this is the behaviour suggested by the feedback itself.

Player activity may be considered an indicator for social presence in mediated communication [1], and for a social game such as Magpies, additional socio-contextual information serves to both add an additional layer of context onto the social gaming experience, and also to make the gaming experience more engaging for the players.

We have demonstrated that adding rich socio-contextual information shown to game players can enhance their general engagement with the game. However, it is not clear what specific contextual information is the most effective. In Magpies, players had a large selection of indices to browse, but the relative efficacy of each index is unknown.

\section{REFERENCES}

1. Biocca, F., Harms, C. \& Burgoon, J. K. Toward a more robust theory and measure of social presence, Teleoper. Virtual Environ., MIT Press, 2003, 12, 456-480

2. DiMicco, J. M., Pandolfo, A. \& Bender, W. Influencing group participation with a shared display CSCW '04: Proceedings of the 2004 ACM conference on Computer supported cooperative work, 614-623

3. Erickson, T. and Kellogg, W. A. (2000) Social translucence: an approach to designing systems that support social processes. ACM Trans. Comput.-Hum. Interact. 7, 1, 59-83.

4. Gjoka, M., Sirivianos, M., Markopoulou, A. \& Yang, X. Poking facebook: characterization of osn applications WOSP '08: Proceedings of the first workshop on Online social networks, ACM, 2008, 31-36

5. Kim, T., Chang, A., Holland, L. \& Pentland, A. Meeting mediator: enhancing group collaboration using sociometric feedback CSCW, 2008, 457-466

6. Kirman, B., Lawson, S., et al, S. Familiars Manipulating Social Networks with Mobile Gaming, Proceedings of Games Design and Technology Workshop and Conference (GDTW) 2008, Liverpool

7. Kluger, A.N. \& DeNisi, A. (1996). The effects of feedback interventions on performance: a historical review, a meta-analysis and a preliminary feedback intervention theory. Psych. Bulletin, 119, 254-284

8. Madan, A. \& Pentland, A. VibeFones: Socially Aware Mobile Phones 10th IEEE Wearable Computers

9. Martino. F., Baù, R., Spagnolli, A., Gamberini, L. (2009). Presence in the age of Social Networks: Augmenting Mediated Environments with Feedback on Group Activity. Virtual Reality, 13 (3)

10.Martino, F., Miotto, A., Davide, F. and Gamberini, L, Exploring Social Network Indices as Cues to Augment 
Communication, $1^{\text {st }}$ International Workshop on Maps

Based Interaction in Social Networks (MapISNet) 2007

11.Nazir, A. Raza, S. \& Chuah, C. Unveiling facebook: a measurement study of social network based applications IMC '08: Proceedings of the 8th ACM SIGCOMM conference on Internet measurement, ACM, 2008, 43-56

12.Rogers, E. M., Diffusion of Innovations, $5^{\text {th }}$ Edition, Free Press, 2003
13.Steinkuehler, C. \& Williams, D. Where Everybody Knows Your (Screen) Name: Online Games as "Third Places" J of Computer-Mediated Comm, 2006, 11

14.Wasserman, S. \& Faust, K. Social Network Analysis: Methods and Applications, New York: Cambridge University Press, 1994 